\title{
FLIES AND TYPHOID.
}

\author{
By WILLIAM NIOOLL, M.A., D.Sc., M.D., D.P.H. \\ Late Ernest Hart Memorial Scholar of the British Medical \\ Association, and Grocers' Company Research Scholar. \\ (Australian Institute of Tropical Medicine.)
}

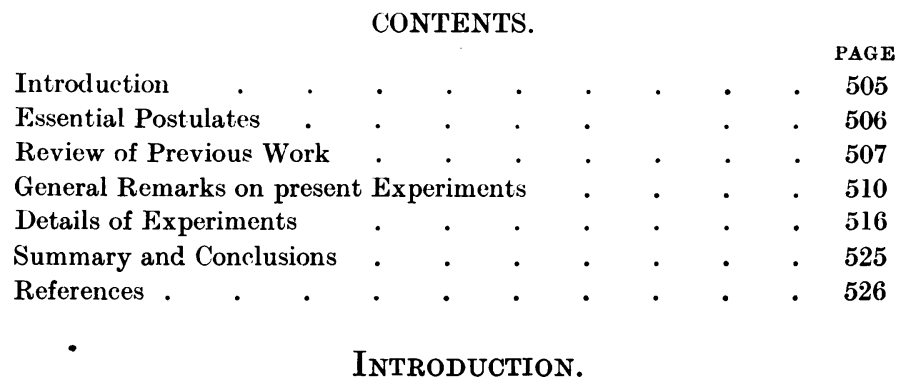

THE relation of insects to human disease has engaged a very large share of attention in recent medical research. Fleas, lice, bugs, mosquitoes and flies have severally been incriminated as carriers of infection. In every case, more or less evidence, drawn both from experiment and from observation, has been adduced to establish a case for or against, but in every instance there remains a modicum of knowledge which is still denied us. Thus, although it is an almost universal canon of scientific belief that fleas transmit plague, that certain mosquitoes transmit malaria, others yellow fever and that tsetse flies are the transmitters of sleeping sickness, yet there are numberless points on which our knowledge is by no means clear or satisfactory. It is now almost universally admitted that the common house fly, and perhaps other flies, play a certain though not extensive part in the transmission of typhoid fever. To an impartial mind it must be apparent, however, that the weight of evidence in this particular case is largely circumstantial. Although the amount of experimental work is by no means inconsiderable it must be admitted that it is not at all conclusive or convincing. 
The best known and most exhaustive work on this matter is that of Graham Smith (1913). The work, however, is open to the same objection as all experimental work of the same type, namely, that it is essentially artificial and does not entirely reproduce natural conditions.

On summarising the fairly voluminous literature, as Graham Smith has done, one finds that apart from the work of that author himself there has been no extensive systematic work on the subject. Most of the contributions to the chain of evidence have been somewhat scrappy. This, however, is to a large extent in the nature of the problem and it is possible that the extensive researches of Graham Smith do not, perhaps, constitute the most important links in the chain.

\section{The Essential Postulates.}

It may be well to analyse the postulates necessary to incriminate the house fly as a transmitter or disseminator of typhoid fever.

It is essential that:

1. Persons infected with typhoid bacilli should provide material which may come into contact with house flies in some way or other.

2. This material should contain virulent typhoid bacilli.

3. These bacilli should be taken up by the flies from this material.

4. The bacilli should be able to exist and retain their virulence on or in the fly for a certain length of time.

5. The fly should be able to convey these bacilli to some other person or persons.

6. The bacilli when so transmitted should retain their virulence and be able to reproduce the infection.

The first postulate is satisfied by the fact that human excreta, particularly faeces, attract the common house fly both for feeding and for breeding purposes. The excreta, not only from patients suffering from typhoid fever, but also from "typhoid carriers" have been shown to contain typhoid bacilli which have not lost their virulence, a fact which satisfies the second postulate.

With regard to the third postulate the evidence is by no means conclusive. Graham Smith has shown that house flies can ingest typhoid bacilli from pure cultures and from experimentally infected material. Faichnie, alone, has shown that flies can ingest and retain B. typhosus from human excreta under natural conditions but his results are so remarkable that some confirmation is necessary. 
The fourth and fifth postulates have by no means been satisfied. It is true that typhoid bacilli have been shown to be capable of surviving in flies for a short time when ingested in pure culture and there is no doubt that these flies can convey the bacilli to articles which furnish a ready means for further infection. It has not, however, been proved that such bacilli retain their virulence and can cause the onset of typhoid fever.

It is evident then that the weak links in the chain of evidence incriminating the house-fly as a disseminator of typhoid fever are:

1. The ability of the fly to ingest bacilli from natural sources in sufficient number.

2. Its ability to carry such bacilli living for a sufficiently long period, and

3. The ability of the bacilli to retain their virulence when deposited by the fly on materials likely to be consumed or handled by human beings.

Very little experimental work has been done towards strengthening any of these three links and it has therefore been deemed advisable to publish here the results of some experiments which may add at least a slight degree of strength to the first of them.

No mention has been made in the foregoing remarks of observational work in the field, i.e. in military encampments. Many observations have now been made in various campaigns and these, without exception, serve to incriminate the domestic fly as a frequent disseminator of typhoid fever: but the evidence, so derived, is, in the main, circumstantial and it yet lacks the support of careful experimental work.

The experiments about to be described were originally intended to cover the whole field of the subject, but they were interrupted at an early stage and no further opportunity of resuming the work has offered itself. It has therefore seemed desirable to publish the results in spite of their incomplete nature.

\section{Review of Previous Work.}

It may be well, here, to make a few brief remarks on the observations and conclusions of Graham Smith and other workers in so far as they concern the present experiments. He remarks (1913, p. 6) "The difficulties attending the isolation and identification of pathogenic bacteria, particularly those belonging to the typhoid-colon group, from 'wild' flies are especially great, since allied, almost indistinguishable types are frequently present in the intestine of flies. No 
diagnosis should therefore be accepted unless all the known tests for identification have been applied." This comment is applicable to the work of earlier observers and to that of some later workers who have not always been able, or troubled, to establish thoroughly the identity of the bacteria isolated by them.

With regard to Faichnie's work the objection is that it does not seem absolutely certain that he was actually transmitting the typhoid bacilli through the larvae to the adult flies, but was really allowing the emerging flies to contaminate themselves from the faeces to which they had access for a considerable time after emerging. The fact, however, that he submitted the bacteria recovered from the flies to independent sero diagnosis proves that he undoubtedly recovered $B$. typhosus. As will be shown presently, however, the probability is that the bacilli were not transmitted through the larvae to the adults, but were picked up by the adults themselves after emergence. The fact that he was able to recover $B$. typhosus after as long a period as 16 days is remarkable and does not accord with the experience of any other observer.

Opposed to Faichnie's assumed results are the experiments carried out by Graham Smith (1911) with the larvae of blow-flies (Calliphora erythrocephala) and $B$. typhosus and $B$. enteritidis. These experiments were entirely negative. Ledingham (1911) working with Musca domestica and $B$. typhosus had a negative experience similar to that of Graham Smith, until he adopted the plan of sterilising the external surface of the eggs and feeding the resulting larvae on large doses of pure cultures of $B$. typhosus. Under these extremely unnatural conditions typhoid bacilli were recovered from the larvae and from one pupa. Unfortunately no adult flies were hatched out. These experiments as already mentioned were extremely unnatural but were presumably the most favourable for the transmission of $B$. typhosus from larva to adult.

Ledingham also remarked on the frequent occurrence of an organism which he designates Bacillus $A$ and which resembles $B$. typhosus on plate cultures, but differs from it in turning litmus milk alkaline, in producing indol and in not fermenting sorbite. Graham Smith (1913, p. 117) refers this bacillus to his type Ca 8 but in my opinion it should really be designated $\mathrm{Ca} 12$ on account of the A-Alk. with reaction. A bacillus which I have frequently isolated from flies closely resembled Ledingham's bacillus A but differed in being non-motile and in fermenting sorbite slowly, approaching in these respects more closely to Graham Smith's type Ch 12, but being non-motile would correspond 
to the vacant place $\mathrm{Ch} 11$, or, if it fermented salicin, to $\mathrm{Cf} 11$. It is to be noted that the fermentation of sorbite was slow and never occurred within 24 hours.

Nicholls (1912) has also remarked that B. typhosus disappeared quickly from larvae of Sarcophaga.

As already remarked Graham Smith (1912) was unable to recover $B$. enteritidis from blow-flies, the larvae of which had been fed on meat contaminated with that bacillus. He showed, however, that many nonlactose fermenters, such as Morgan's bacillus No. 1, will survive from the larva to the adult.

Tebbutt's results (1913) are also in opposition to those of Faichnie. He found that $B$. dysenteriae could not be recovered from pupae or adults, the larvae of which had been fed on that organism, except when the eggs were previously sterilised. B. typhosus, however, could not be recovered at all.

The whole subject of the transmission of bacteria from larval to adult flies is ably and briefly summed up by Graham Smith (1913, pp. 123-124) but I venture to think he is inclined to stretch a point, in endeavouring to account for the results obtained by Faichnie, by drawing the distinction, which every bacteriologist recognises, between the behaviour of cultivated and uncultivated or natural strains of the same organism. The matter, however, is one which cannot be ignored and it forms to a certain extent the raison d'être of the present paper. Graham Smith refers to the same subject again on p. 125 and his remarks there are worthy of the fullest consideration.

In dealing more particularly with the subject of flies and B.typhosus, Graham Smith (1913, p. 129) again refers to the difficulty of isolating B. typhosus from the intestine of the fly. His remarks are practically a paraphrase of those quoted above. He adds, however, "The occurrence of these non-lactose fermenting bacilli probably often leads to failure in isolating $B$. typhosus even when it is present, and in any case renders its detection a long and laborious undertaking." It might be added that not infrequently the reverse might be the case and organisms may be identified as $B$. typhosus which are not really so.

Ficker (1903) was able to isolate $B$. typhosus from artificially infected flies for 5-23 days after infection. In this case in establishing the identity of the organism, agglutination tests were employed. Graham Smith (1910) however, also using artificial infection, could not recover $B$. typhosus later than six days from the intestine and only two days from the faeces. 


\section{General Remarks on Present Experiments.}

The experimental work detailed in the following pages was carried out during my term of the Ernest Hart Memorial Scholarship of the British Medical Association (1909-1912).

Owing to the incompleteness of the results, publication has been delayed in the hope that opportunity would offer for amplification. As this hope has not been fulfilled it seems advisable to publish the results even in their incomplete state.

The experiments were started as a sequel to the work already done on the general bacteriology of Musca domestica (Journal of Hygiene, XI. 1912). The earlier work though essentially preliminary in character showed that a large variety of bacteria belonging to the colon group occurred naturally in flies. Attention was also directed to the occurrence of non-lactose-fermenting bacilli such as $B$. paratyphosus $\mathrm{B}$ and Morgan's bacillus No. 1 in flies in the natural state. No mention, however, was made of a large number of other non-lactose-fermenting bacteria which were isolated and carefully studied as it was impossible to identify them with any known pathogenic or non-pathogenic forms. Their characters presented many difficulties with which, at that time, I did not feel prepared to cope. Since then several authors, particularly Graham Smith (1912), have published descriptions of non-lactosefermenting bacilli, agreeing closely with those met with during the course of my investigations.

Quite a number of the organisms referred to above initially bore a striking resemblance to $B$. typhosus. On neutral red bile salt media they were indistinguishable from colonies of $B$. typhosus except that they appeared a trifle brownish after 24 hours incubation at $37^{\circ} \mathrm{C}$. It was discovered later however that the same colonies would appear when the plates were incubated at room temperature $\left(15^{\circ}-20^{\circ} \mathrm{C}\right.$.). Hundreds of these colonies were sub-cultured in fermentable media and in a large number of cases gave, for the first few days, reactions identical with that of $B$. typhosus. In every case agglutination tests were made with stock typhoid serum and in the large majority of cases the result was unequivocally negative. In the few doubtful cases the agglutination tests were repeated and absorption tests were also employed. For help in these tests I am indebted to Drs Bainbridge, Ledingham and Petrie, who made independent controls.

In every case the results were negative and this was confirmed by the fact that on the fourth to the sixth day the cultures began to 
ferment lactose. By the sixth day in the great majority of cases the lactose broth showed a distinct reddish colour and in the few other cases the appearance of the red colour was not delayed beyond the tenth day.

The foregoing remarks apply only to organisms apparently resembling those of the typhoid group. Numerous other non-lactose fermenters were isolated resembling essentially $B$. paratyphosus $\mathrm{B}$ and Morgan's bacillus No. 1 but differing from them in one or more important details. It is worthy of remark here that no organisms resembling $B$. paratyphosus A or $B$. enteritidis Gaertner were ever isolated from the flies I examined.

It should be noted that the preliminary methods of identification employed in these investigations were those adopted by Morgan and Ledingham (1909) and by Graham Smith (1912).

The bacilli mentioned in the foregoing paragraphs will be dealt with in more detail in the second part of this paper. . The more immediate object of the present communication is to discuss the results of experiments in which there could be no question that $B$. typhosus was actually involved.

The particular experiments on $B$. typhosus were commenced in June, 1911, and continued till February, 1912. The flies used were entirely Musca domestica, the majority being flies reared from larvae in captivity, the others being "wild" flies captured in the warmer parts of the Institute. It may be remarked that no flies were taken from any quarter within a radius of 80 yards of any place where typhoid experiments were being undertaken. As a matter of fact the only two places in which B. typhosus was present were the laboratory of the chief bacteriologist and the stock culture room, both more than 20 yards from the experimental room and neither likely to contain exposed cultures of $B$. typhosus. The infective material was obtained from Dr Ledingham, Chief Bacteriologist at the Lister Institute, and consisted of the faeces of suspected typhoid carriers, which Dr Ledingham was systematically examining for the Local Government Board. In every case the faeces sent up to me were from cases which had proved to be undoubted typhoid carriers, but the particular sample submitted to me was not guaranteed to contain $B$. typhosus.

On the material thus obtained batches of flies were fed for periods varying from 20 to 60 minutes. From the material cultures were made and found to contain $B$. typhosus in every case except two. These findings were later confirmed by the report from the laboratory of the chief bacteriologist. 
After the flies had been allowed to feed on the infected material, it was removed and they remained for a longer or shorter period without food. The members of each batch of flies were examined over a period of 48 hours. Each batch of flies consisted of about half a dozen, which from previous experience had been found the most convenient number to deal with. In the first seven batches of flies the results, so far as $B$. typhosus was concerned, were entirely negative. In the eighth series of seven flies one was discovered to harbour $B$. typhosus 28 hours after feeding. The next five batches were entirely negative. The fifteenth showed one fly infected after 5 hours and another after 14 hours. The next three batches were negative. The nineteenth gave the most favourable result. One fly showed infection 45 hours after feeding while another gave a culture 36 hours after feeding. Of the remaining seven batches only two were positive and both under 24 hours. One gave a positive result in 23 hours and the other in 9 hours. Thus out of a total number of 163 flies used only 7 gave a positive result.

It is thus evident that the chance of flies becoming infected from infective faeces under these experimental conditions is not very large and under natural conditions the chance must be very considerably less.

The experiments were conducted for the most part in sterilised glass chimneys, closed at the top end by a layer of muslin and at the bottom by a glass plate. Several, however, were conducted in a specially constructed cage described in a previous paper (Nicoll, 1912). Each batch consisted of 7 to 8 flies which were allowed to feed on the infected faeces for a period of from 4 to 24 hours. The faeces was then replaced by sterilised sugar and water. A 1 per cent. solution of cane sugar in distilled water was used. After the lapse of about an hour one fly was removed and examined. The procedure was similar to that already described (Nicoll, 1912). The fly was first thoroughly washed in peptone broth which was thereafter incubated at $37^{\circ} \mathrm{C}$. It was then washed in sterile water and then immersed for 10 to 20 minutes in absolute alcohol. After being dried over a flame it was again washed in broth which was incubated as a control. The fly was then dissected and the alimentary canal teased up in broth and incubated. In several cases the various parts of the fly were incubated in separate tubes of broth.

The broths were incubated for 12 to 18 hours, then plated on neutralred bile salt agar. From these plates all white colonies were picked off, or in cases showing an excessive number of white colonies about 
ten or a dozen were selected. Each colony was again incubated in peptone broth and replated. The pure cultures so obtained were submitted to the usual tests.

The remaining flies of each batch were examined at intervals, and at the conclusion of the experiment cultures were made from the sugar and water and from the vomit and faecal marks left on the glass chimneys and plates.

The tests used in identification were the fermentative reactions in lactose, glucose, mannitol, saccharose, dulcite, adonite, sorbite and litmus milk together with the motility, production of indol and agglutination with stock typhoid serum.

A fact which is probably of little significance, but none the less worthy of note is that there was a comparatively high mortality amongst the flies used in the experiments. This was most noticeable in the winter months (December to February) but was also remarked on during the summer and autumn while other experiments, in which flies were fed on faeces from various sources, were being conducted. It may be a matter of mere coincidence, but the observation is suggestive of the possibility that these faeces contained something deleterious to the flies.

In the course of the experiments 1062 colonies were examined, 578 of those proved to be colonies having little in common with the typhoid and allied groups of bacilli. Of the remaining 484 colonies, 361 did not ferment mannite. There remained a total of 123 which appeared to have some relation to $B$. typhosus ; 98 of those, however, were nonmotile organisms which eventually fermented lactose (in 4 to 6 days). Thus only 25 of the 1062 colonies proved to be B.typhosus, and these as already mentioned were obtained from 7 flies.

As was to be expected, in most cases more than one colony of the same type was picked off from one plate. These duplicates except in the case of $B$. typhosus were discarded and there remained 357 separate cultures. 25 of these, as already mentioned, were B. typhosus. The other 332 cultures were found to comprise 49 different varieties of bacilli. On an average, therefore, on every fly examined there were at least two varieties of non-lactose-fermenting bacilli. As mentioned above a considerable percentage of these proved to be in reality slow lactose fermenters and they caused considerable trouble in the course of the investigations.

Throughout the course of the experiments four days was taken as the critical period for the fermentative reactions though actually the 
period was $3 \frac{1}{2}$ days, as the media were usually incubated in the afternoon and examined on the morning of the fourth day. As a matter of fact in the vast majority of cases constant results were obtained on the morning of the second day (i.e. after about 42 hours). In.every case, however, the media were kept up till the tenth day, but changes after the sixth day (morning) were neglected; except in the case of litmus milk which was kept for fifteen days.

Reference has already been made to the frequent presence of bacilli resembling $B$. typhosus in all their fermentative reactions, and the indol reaction. The action in litmus milk too, as shown later, was identical yet the organisms were invariably non-motile and did not agglutinate with typhoid serum. The investigation of these bacilli consumed a considerable amount of time and resulted in the conclusion that organisms of this type flourish in flies. This observation finds confirmation in the work of Ledingham and Graham Smith who frequently found bacilli identical with those met with in my experiments. These independent findings cannot fail to raise the question of the occurrence of a specific bacterial flora in the intestine of the house fly. I have frequently obtained almost pure cultures of bacilli of this type from the intestine of flies, and the fact causes one to pause in estimating the results of earlier investigations. It cannot be doubted that many of these organisms might readily be mistaken for B. typhosus, and even such an experienced bacteriologist as Graham Smith expressed his uncertainty when he first encountered them.

It is a curious fact too that Ledingham in the course of his massiveinfection experiments with fly larvae and B. typhosus cultures should have encountered this type of bacillus so frequently. These results from well accredited sources throw very great doubt on the experimental results of Bahr. On the other hand it might be assumed that Bahr is correct and that bacteriologists such as Ledingham and Graham Smith have not grasped the true facts of the case, namely that $B$. typhosus and other organisms have been transformed in the intestine of the fly or its larvae into bacilli of a somewhat different type. On this matter or indeed on the transmissibility of any species of organism, be it bacteria, plant or animal, I have an open mind but my results in the present instance endorse the caution of Graham Smith and the almost uncompromising negativeness of Ledingham.

As an addendum to the foregoing it may be remembered that I have conducted experiments with a view to determining what might be considered the natural intestinal flora of flies. These experiments 
were unfortunately not entirely satisfactory as a number of discrepant results were obtained. On the whole, however, they showed several interesting facts. Batches of "wild" flies were captured and enclosed in glass chimneys. They were fed on sterile foods for various periods. Two or three of each batch were examined bacteriologically at the outset and were found to contain an almost overwhelming preponderance of organisms of the $B$. coli type. The remaining flies were fed on sterile food, chiefly water, sugar and bread. It was found that after a week the colon bacilli had very largely disappeared. After 13 to 14 days colon bacilli had absolutely disappeared, except in a few cases. After the eighth day, as a rule, the bacterial flora became almost purely non-lactose or apparently so. There was an absolute absence of bacilli of the typhoid-colon type. After the eighth day the bacterial flora began to display a characteristic non-lactose type. These bacteria were mainly of the type already mentioned above and were in reality not non-lactose fermenters in the strict sense of the term. In addition, however, a considerable number were met with conforming to the type of Morgan's No. 1 bacillus.

With regard to the feeding of flies many observations have already been made. Graham Smith has given a very full and complete account of this matter, and I have added one or two observations which may serve to show that flies are attracted by excrement both from human and other sources. It was pointed out in the previous paper that an essential point was that the excrement should be fresh and moist. When it becomes dry after the lapse of several hours the attraction is very greatly diminished. On that account, in the foregoing experiments it was necessary to moisten the material every 24 hours to start with and eventually twice and three times a day. Naturally after the first 24 hours the freshness had gone and it was only by the added moisture that the flies could be attracted.

From the foregoing paragraph it is evident that flies are attracted chiefly by moist excreta and this, as far as the dispersal of pathogenic bacteria is concerned, seems to be an argument against the disposal of human faeces in shady, sheltered places where they remain moist and fresh for a considerably longer period than if they were exposed to the open air or the direct rays of the sun. From these observations it is obvious that the ordinary field-latrine, dug in a secluded place if possible, is one of the most favourable feeding and breeding grounds for flies. It is, as a rule, sufficiently deep to exclude the sun's direct rays, and from the accumulated urine it is kept in a sufficiently moist 
state to attract flies. It is true instructions are given for the use of sawdust, sand and other absorbent materials, but the chance of those being employed as a routine is extremely remote. This view is derived from experience in civilian centres where a very large percentage of the population ignore such precautionary measures.

\section{Details of Experiments.}

I. June 16. 6 p.m. Six flies enclosed in one section of experimental cage along with normal human faeces impregnated with urine from typhoid patient.

„ 16. 7.30 p.m. Three flies transferred into second section of experimental cage in which some sterile moist bread had been placed.

" 17. 3.30 p.m. Bread removed and cultures made (Culture No. 1). Fresh bread introduced.

„ 17. 4 p.m. One fly from Section II examined. After immersion in absolute alcohol and normal saline, washed in peptone broth (Culture 2). Cultures also made from the intestines (No. 3), crop (4) and salivary glands (5).

, 18. 4 p.m. Cultures made from bread (6); fresh bread introduced.

„ 19. 8 p.m. Cultures made from bread (7). Several flies dissected, cultures made from alimentary canal (8).

„21. 9 a.m. Remaining flies dead. Combined cultures made from their alimentary canal (9).

All these broth cultures were plated on neutral-red bile salt agar, and it was found that Nos. 2 and 8 were entirely sterile, while Nos. 1, 3, 4, 5, 6 and 7 showed only red colonies. No. 9 showed numerous white colonies which, however, were not B. typhosus. No red colonies were present.

II. June 22. 12 noon. Twenty flies put into cage along with faeces from typhoid patient.

„22. 7 p.m. Six flies transferred to second section of cage into which solution of sugar in water introduced (majority of flies in Section $I$ already dead).

, 23. 12 noon.

Two flies dead in Section II; others living. Cultures made as follows:- - exterior of first fly (10) exterior (11) and elementary canal (12) of 2 nd fly; also from intestine of fly in Section I (13) and from sugar solution (14). Fresh water and bread introduced Section II.

,24. 4 p.m.

Bread removed from Section II. Cultures made from it into broth (15) and directly on to bile salt agar (16). All flies in Section II living: only three living in Section I. Cultures made from fly from Section II; external (17) internal, intestine (18) and crop (19). 
June 27. 4 p.m. Cultures made from faeces:-1. Direct on to bile salt agar (20). 2. Into peptone broth (21). Bread removed from Section II and cultures made. Broth cultures (22) and direct plate (23). Emulsion made from dead flies in Section I, cultures (24 and 25): plate (26).

The results of these cultures were as follows:-

No. 10. Showed only cocci.

No. 11. Had cocci and colon bacilli.

Nos. 12 and 13. Only coli.

No. 14. Sterile.

No. 15. Coli, cocci and one white colony which was not B. typhosus.

No. 16. Coli, cocci and some white colonies which were not B. typhosus.

No. 17. Cocci only.

No. 18. Coli and cocci. No white colonies.

No. 19. Sterile.

No. 20. Only coli.

No. 21. Coli and white colonies which produced gas in mannite.

No. 22. No white colonies.

Nos. 23, 24 and 25. Several white colonies which produced gas in mannite.

No. 26. Coli and several white colonies resembling B. typhosus but differing in several reactions.

From this it can be seen that none of the cultures made in this experiment contained B. typhosus. Cultures made from the infected material at the end of the experiment proved to be negative which might be taken as evidence that the typhoid bacilli had all died out.

III. June 27. 5 p.m. Fifteen flies introduced into cage with infected faeces from typhoid carrier, cultures made from faeces $(27,28)$ moist bread placed in Section I.

, 28. 10 a.m. Six flies transferred to Section II into which a piece of moist bread was introduced.

„28. 6 p.m. Cultures made from bread in Section I (29).

" 30. 1 p.m. Cultures again made from bread in Section I, Plate (30) Broth (31). Then flies from Section II examined together. Cultures made from external surface (32) and alimentary canal (33).

, 30. 6 p.m. Cultures made from fly faeces on bread dish in Section I, plate (34) and broth (35).

July 1. 7 p.m. Three flies from Section II examined. Cultures from exterior (36), alimentary canal (37). Cultures made from bread in Section II (38). Cultures also made from faeces (39). Experiment discontinued.

The results of these cultures were

The cultures from the faeces on 27th June showed the presence of B. typhosus, but on lst July the cultures were negative. From the flies only B. coli and cocci were obtained. The bread showed the same organisms, while the cultures made from the fly faeces proved sterile.

Journ. of Hyg. $x \mathrm{v}$ 
IV. July 5. 4 p.m. Thirty flies put into Section I of eage along with moist faeces from typhoid carrier and some fresh bread.

" 6. 4 p.m.

, 7. 11 a.m.

, 8. 4 p.m.
Fourteen flies transferred to Section II. Cultures made from one fly, exterior (40) and alimentary canal (41).

Plate culture made from faeces (42) from bread in Section I (43) and from bread in Section II (44). Four flies from Section II examined, and cultures made from exterior and alimentary canal (45-52).

Ten flies from Section II examined. Cultures 53, 54, 56 and 57. Cultures made from bread in Section II (55) and from bread in Section I (58).

This experiment was more interesting than the previous three owing to the fact that a large proportion of the cultures showed the presence of non-lactosefermenting organisms. The plate cultures made from the typhoid faeces displayed only coliform colonies. The alimentary canal of the flies provided in every case almost pure cultures of non-lactose-fermenting bacilli, all of which either did not ferment mannite or produced gas on that medium. The exterior of the flies provided a more varied flora of colon bacilli, cocci and occasional non-lactose bacilli. The cultures from the bread showed in every case non-lactose bacilli which fermented mannite with gas production. B. typhosus was not isolated in any case.

V. On July 9th a fresh sample of faeces from a typhoid carrier was placed in the cage along with the flies left over from the foregoing experiment. On the 9th fifteen flies were transferred from Section I to II. Five hours later four of these flies were dissected and cultures were made from their external surface and their alimentary canal. In every case non-lactose organisms were isolated from the intestine and in one case from the external surface. In three instances, the organisms so isolated did not ferment mannite, while the other two fermented it with gas formation. Twenty-four hours later another three flies were examined with similar results. The following day (July 11th) four flies were examined en masse. From the external surface several non-lactose colonies were isolated and these either did not ferment mannite or produced gas on that medium. From the intestine numerous non-lactose colonies were isolated. The majority of these produced gas on mannite but a few fermented that medium without the production of gas. In many other respects these organisms resembled B. typhosus but they fermented saccharose. Several cultures were tested with typhoid serum but all failed to agglutinate. A few hours later another two flies were examined in a similar fashion and gave results identical with the previous batch. On the same day two lots of five flies each, from Section I, were examined. In both cases almost pure cultures of non-lactose-fermenting organisms were isolated, but these proved to be either non-mannite or mannite fermenters with gas production. On July 12th another batch of four flies from Section I was examined and gave results similar to the previous lots. Two days later a further lot of four flies from Section I was examined. From the external surface of these an almost pure culture of non-lactose organisms producing gas on mannite was obtained, while from the intestine a pure culture of non-lactose, non-mannite colonies was obtained. 
At the same time two flies from Section II were examined with similar results. Two days later another two flies from Section I were examined and again both results were similar to the foregoing. Three days later the experiment was concluded. Ten flies were left in Section I. These were divided into three lots of two, four and four respectively. From the external surface of the first lot several lactose-fermenting organisms were obtained, while from the intestine an almost pure culture of a non-lactose, mannite fermenting bacillus was obtained. This bacillus, however, fermented saccharose and liquefied gelatine. From the second lot an almost pure culture of non-lactose, non-mannite bacilli was obtained. These organisms fermented glucose with gas formation. From the last lot the organisms isolated were chiefly non-lactose with gas on mannite.

This experiment is interesting in several respects. As far as B. typhosus is concerned it was entirely negative, of that there is no question. From start to finish the last batch of flies was in captivity for a fortnight. The time of the year-the middle of summer-was that most favourable for the life of flies and as has been stated in a report on previous experiments flies can be kept alive and in healthy condition in captivity for at least six weeks. My endeavour throughout the experiment was to keep the flies in a healthy condition.

Fresh water passed through a Berkefeld filter and moist bread, boiled in water for ten minutes, were supplied to them every day or as occasion required. The most remarkable result is the disappearance of the lactose-fermenting organisms of the $B . c o l i$ type and their replacement by non-lactose-fermenting organisms. This curious phenomenon has been referred to in the paper mentioned above. These organisms fell naturally into three groups according to their behaviour on mannite, namely non-fermentation, fermentation without gas production and fermentation with gas. In the first category the organisms were mainly of the type of Morgan Nos. 1 and 2, though a few were found corresponding to type V. In the second category three of the types (Flexner) designated by Morgan and Ledingham (1909) occurred along with a few others not included in their list. In the third category the majority of the organisms isolated conformed to type No. $\mathrm{X}$ of the above classification, but a few were also found of the type XII and XIV.

VI-VIII. During September three further sets of experiments were conducted on the same lines. In each case the results were entirely negative.

IX-XI. During October another three sets of experiments were carried out. At this time the flies began to be attacked by the fungus, Empusa muscae, and the mortality from this cause was 30 to 40 per cent. The technique in these experiments was slightly modified. The various parts of the flies were lightly mashed up in broth and placed in the incubator at $37^{\circ} \mathrm{C}$. for four hours. Thereafter the emulsions were thoroughly ground up and cultures made in broth or direct on bile salt agar. Forty-seven flies were used in these experiments and in every case the result was negative.

XII. On November lst a different experiment was tried. A culture of B. typhosus was made from the faeces of a typhoid carrier (C. R.) and a sub-culture of this was made on plain agar. From this an emulsion was prepared and placed along with some sugar in a vessel into which five healthy flies were introduced. Seven hours later two flies were examined and from their legs a pure culture of typhoid bacilli 
was obtained. This was identified by all the possible tests except inoculation. From the intestine and crop three different white colonies were obtained. One of these resembled $B$. typhosus except that it was non-motile and produced no reaction on litmus milk. Of the other two varieties one gave an alkaline reaction on milk and produced indol, while the other fermented saccharose and was nonmotile. None of these strains would agglutinate with typhoid serum even in low dilution. Cultures were made from the faecal marks left by the flies. Here again white colonies were obtained but they were non-motile and fermented lactose after six days. Cultures from the vomit marks were negative.

The flies were then transferred to a clean vessel and after 24 hours the faecal and vomit deposits were again cultured with negative results. On November 2nd the flies were again transferred to a third vessel and allowed to remain in it for nearly ten days. At the end of 43 hours cultures were made from the faecal deposits which were negative. Cultures from the vomit marks, which were almost solid deposits of sugar, gave a growth of almost pure B. typhosus. The bacilli gave an agglutination of $\mathbf{I}$ in 2000 with typhoid serum.

Five hours later a single fly from this batch was examined: the legs and proboscis gave a negative result but from the intestine several colonies were isolated which proved to be $B$. typhosus, giving an agglutination of 1 in 2000 with . typhoid serum. The remaining flies of this batch were examined, one every day, but gave a consistently negative result. The faecal and vomit deposits were also examined but they, too, were negative.

XIII. On November 2nd six flies were placed in a vessel with faeces from a typhoid carrier. They were allowed to remain undisturbed till November 5th when one fly was examined. Cultures from its legs gave only $B$. coli. From the intestine numerous white colonies were obtained, but they all proved to be slow lactose fermenters. Two days later another fly was examined, and both from the external surface and from the intestine white colonies were obtained. In every case, however, they were non-motile organisms and did not ferment mannite. On the following day the last living fly was examined. From its legs only cultures of cocci were obtained but from its intestine numerous colonies of a motile bacillus were obtained. From the sugar reactions these organisms appeared to be $B$. typhosus but they produced indol, and on litmus milk they gave, at first, an acid reaction but eventually an alkaline one. On being tested with typhoid serum there was no agglutination.

XIV. On November 4th six flies were placed in a vessel with faeces from the same typhoid carrier. Five hours later cultures were made from the faeces. No typhoid bacilli were obtained. All the organisms isolated were colon bacilli. At the same time a single fly was examined. Nothing of note was found on the legs or exterior but from the intestine numerous non-lactose colonies were obtained. On further investigation these were identified as Morgan's bacillus No. 1. Next day another fly was examined. From its legs an almost pure culture of a nonlactose-fermenting organism was obtained. This resembled Morgan's bacillus, but its identity was not absolutely established. From the intestine, on the other hand, a pure culture of a non-lactose-fermenting bacillus was obtained. This resembled $B$. typhosus in all its reactions except on litmus milk which it turned 
from acid to alkaline on the third day. On being tested with typhoid serum it gave a negative result (1/1000 with a serum of high titre). Two days later another fly was examined. Cultures from its legs were sterile. From the intestine numerous colonies of a slow lactose-fermenting bacillus were obtained. This was motile and fermented glucose, mannite and saccharose slowly. It produced no indol and turned litmus milk permanently acid, but produced no clot. Several non-mannite colonies were also isolated.

On November 8th another fly was examined. From its legs an almost pure culture of $B$. paratyphosus B was obtained. This organism agreed in all its cultural reactions with those of $B$. paratyphosus B except that it produced very little gas on mannite. It agglutinated with paratyphoid serum up to 1 in 500. From the intestine and crop of the same fly cultures were made but these proved sterile.

On November 9th another fly was examined, from its external surface nothing but non-lactose colonies were plated, these proved to be Morgan's No. 1 bacillus.

On November 10th another fly was examined. The external surface yielded no growth and the intestine gave only cocci.

On November 11th the last fly of this batch was killed and examined. From the legs and the intestine almost pure cultures of a non-lactose organism were obtained. These again proved to be identical with B. paratyphosus B and they agglutinated with paratyphoid serum to 1 in 500 .

XV. On November 7 th three flies were placed along with faeces from a typhoid carrier (J. C. 5). Cultures were made from the faeces but no B. typhosus were isolated. Eighteen hours later the faeces were found to be dried up and a fresh lot was introduced. Four hours later one fly was examined. From the external surface nothing was obtained, but from the intestine an organism closely resembling B. typhosus was obtained in large numbers. It produced indol, however, and gave an acid to alkaline reaction on litmus milk. It probably corresponds to Morgan and Ledingham's bacillus No. $4 \mathrm{~B}$. At the same time cultures were made from the faecal and vomit marks made by the flies. Neither of these yielded anything.

On the 9th a second fly was examined. The external surface was quite sterile and the intestine yielded only a few cocci and lactose fermenters.

On November 12th the last fly was examined and yielded only lactose fermenters both internally and externally. The faecal marks from the vessel were examined but proved negative.

XVI. On November 7 th five flies were put along with faeces from a typhoid carrier (C. R. 7. XI.). Twenty hours later one fly was examined. From the external surface only cocci were obtained. From the intestine a bacillus was obtained which was motile, did not ferment lactose on mannite but produced acid and gas on glucose and saccharose. It produced indol and turned litmus milk slightly acid. A second fly gave only lactose fermenters and cocci.

Next day a third fly was examined. From the legs only lactose fermenters were isolated. From the intestine a few non-lactose colonies giving acid and gas on mannite were isolated but these were not further identified. From the faecal marks some non-mannite colonies were isolated but not further examined, while from the vomit marks nothing was obtained. 
On the 10th another fly produced only cocci hoth externally and internally. The faecal and vomit marks were sterile. Two days later a dead fly was examined. From the external surface an almost pure culture of a non-lactose bacillus was obtained. This gave acid and gas on glucose and mannite, was non-motile and did not produce indol. The milk reaction was acid to alkaline. From the intestine a different type was isolated. This resembled Morgan and Ledingham's No. 3, except that it turned litmus milk from slightly acid to slightly alkaline. The change, however, was so very slight that it might reasonably be ignored.

On November 14th the remaining flies were dead. One was examined. From the legs no growth was obtained and from the intestine only lactose fermenters were isolated.

XVII. On November 10th five flies were placed along with some faeces (Macconachie 5. XI.). Seven hours later one fly was examined. In the cultures from the legs all the colonies were lactose fermenters. From the intestine a number of slow lactose fermenters were obtained. These fermented mannite and glucose with gas production but did not affect saccharose or dulcite. They gave an alkaline reaction on litmus milk, later turning slightly acid. They were not further investigated.

On November 11th another fly was examined. From its external surface a number of apparently non-lactose organisms were isolated. They fermented both glucose and mannite and after five days they gave a slight acid reaction on lactose and saccharose. On litmus milk they gave a distinct acid reaction all through. From the intestine a large number of white colonies were obtained but none of them fermented mannite and they were accordingly discarded. The faecal and vomit marks were examined. From the former only lactose fermenters were obtained while the latter was sterile.

Next day another fly yielded, on its legs, only lactose-fermenting colonies while the intestine produced white colonies which fermented mannite and glucose with gas production, were non-motile, produced no indol and gave an alkaline reaction on litmus milk.

Ten days later cultures were made from the faecal and vomit marks, both of which were sterile.

On November 16th a third fly was examined, its legs proved to be sterile, but in the intestine an almost pure culture of a non-lactose fermenter was present. This gave acid and gas on mannite, glucose and saccharose, was motile, did not produce indol and gave an acid to alkaline reaction on litmus milk.

Three days later (Nov. 19th) another fly was examined. From its legs only cocci were obtained while from the intestine a single red colony was obtained. Four days later (22nd) the last fly, which was still living, was examined. Neither from its legs nor intestine were any organisms obtained.

XVIII. On November 20th a six hours' broth culture was made from the faeces of a typhoid carrier (Simpson). Four flies were allowed to feed on this. Five hours later one fly had fallen into the culture. It was killed and examined. The external cultures were discurded. From the intestine a large number of white colonies were isolated. On further examination these proved to be B. typhosus. The serum reaction confirmed the diagnosis. Next day another fly was examined. From its legs several non-lactose fermenters were isolated. These differed from B. typhosus 
only in the fact that they produced indol and turned litmus milk alkaline. From the intestine a single red colony was obtained, together with several whites. The latter were non-motile and produced acid and gas on glucose, mannite and saccharose. They produced indol and on litmus milk gave an acid to alkaline reaction. From the vomit deposits pure cultures of $B$. typhosus were obtained.

Three days later the vomit marks were again examined, but proved sterile. After another two days the vomit and the faecal marks were examined but proved negative. Three days later all the flies were dead, one was examined but produced only cocci both internally and externally. The other flies were not examined.

From these foregoing eighteen experiments it is evident that the task of isolating B. typhosus from flies exposed to contamination is by no means an easy one. Only in the last experiment where flies were allowed access to a pure broth culture were typhoid bacilli obtained from the flies. In almost every other case non-lactose fermenters were isolated, some of which appeared to resemble B. typhosus but on careful examination were found to be distinctly different organisms. Again a number of the organisms isolated appeared to be identical with $B$. paratyphosus $\mathbf{B}$ and Morgan's bacillus No. 1 , both of which are regarded as pathogenic in some degree.

The other non-lactose colonies isolated were referable to some extent to organisms isolated by Morgan and Ledingham and by Graham Smith, but a number appeared to be different to any which these authors succeeded in isolating. Again there were a large number of slow lactose-fermenting organisms which closely resembled B. typhosus except in their inability to agglutinate with typhoid serum. As far as I can gather these have not previously been referred to by workers on this subject.

XIX. On November 29th four flies were placed along with faeces from Mrs Brassell (25. xI. 12). Twenty-six hours later the faeces were removed. One fly was killed and examined. From the external surface a number of non-lactosefermenting organisms were isolated none of which proved to be B. typhosus. From the alimentary canal, however, a large growth of non-lactose-fermenting colonies were obtained. The crop and the intestine were examined separately. From the former an almost pure culture of B. typhosus was obtained. These organisms gave all the typical cultural reactions of $B$. typhosus and in addition they agglutinated with typhoid serum up to 1 in 2000 . From the intestine numerous colonies of $B$. typhosus were isolated. Along with them, however, were several other nonlactose colonies. Most of these resembled B. typhosus except that they were nonmotile. The others differed in not fermenting sorbite and in turning litmus milk from acid to alkaline. The faecal matter from this batch of flies was examined and found to contain B. typhosus together with other non-lactose fermenters which fermented glucose, mannite and saccharose with gas production but did not ferment sorbite. They were motile but gave no indol. They probably corresponded to Morgan and Ledingham's type XII 12, as their litmus milk reaction was A.A.A.C. From the vomit marks nothing was obtained.

After another twenty-six hours a second fly was examined. Cultures from its legs produced a few white colonies which were not B. typhosus. After being thoroughly sterilised the fly was mashed up in sterile broth and plates made directly from the emulsion. On these several colonies of B. typhosus were found, these gave a positive agglutination up to 1 in 2000 . The faecal and vomit marks from the 
flies were again examined. The former yielded only a few lactose fermenters, while the latter was negative. Next day the third fly was examined. From its legs numerous non-lactose colonies were obtained which proved to be identical with Morgan and Ledingham's type 4 B. From the intestine several non-mannite, nonglucose bacilli were isolated, but were not further identified. B. typhosus was not recovered. The last fly of this batch was found dead next day and as it was dried up it was not examined.

$\mathrm{XX}$. On November 30th nine flies were placed along with faeces from C. R. (29. xr.). Twenty-five hours later the faeces were removed. From the legs of one fly numerous non-lactose colonies were isolated. These proved to be identical with type $4 \mathrm{~B}$ (Morgan and Ledingham) in all their cultural reactions. From the intestine only some non-mannite fermenters were isolated. From the faecal marks left by the flies Bacillus $4 \mathrm{~B}$ was again isolated in considerable numbers. The vomit marks yielded only non-mannite fermenters. A second fly examined twentyfour hours later yielded an exactly similar result, Bacillus $4 \mathrm{~B}$ being found on the legs and in the faecal deposits. The same organism was isolated thirty hours later from a third fly, this time both from the legs and the intestine. Two days later all the flies were dead. They were examined in two batches. From the legs of the first an almost pure culture of an organism resembling type 3 (Morgan and Ledingham) was obtained. These fermented lactose slightly after six days. . From the intestine nothing of interest was obtained. From the legs of the second batch what was probably an organism identical with that just mentioned was obtained. The intestine again yielded nothing of interest.

XXI. On December 5th ten flies were put along with faeces from Mrs B. which were heavily charged with $B$. typhosus. Twenty-five hours later the faeces were removed and sugar solutions substituted. By this time two flies had already died. One living fly was examined but was accidentally immersed in chloroform. No growth was obtained from it. Twenty-three hours later four of the remaining flies were dead and one was dying. These were all examined. From the external surface only a few non-lactose colonies were obtained. These proved to be chiefly non-mannite fermenters with a few which produced acid and gas on mannite. From the intestine only lactose-fermenting organisms were obtained. The faecal and vomit marks were examined. The former yielded only cocci and one coliform bacillus, while the latter gave nothing.

The remaining two flies were transferred to a fresh vessel. Two days later one was examined, but yielded only a few non-mannite bacilli both externally and internally. Three days later the last fly was examined. Only a few cocci were obtained from it.

XXII. On December 13th eight flies were placed with faeces from C. R. On examination no B. typhosus could be found in the faeces, but there was a plentiful growth of a bacillus which produced white colonies on MacConkey's medium greatly resembling that of $B$. typhosus. Its cultural reactions resembled those of type 3 (Morgan and Ledingham). It produced acid on mannite, glucose and sorbite but not on saccharose. It was non-motile and gave a strong indol reaction. On litmus milk it gave an acid to alkaline reaction and on lactose it gave a faintly acid reaction after six days. 
Four hours later the faeces were removed and replaced by sugar and water. On examination one fly yielded the above described bacillus in almost pure culture both on legs and in intestine. A second fly yielded the same bacillus from its intestine but no growth was obtained from its legs. From a third fly the same bacillus was removed in almost pure culture from legs. crop and intestine.

Next day two flies yielded the same bacillus in great abundance both on the legs and in the alimentary canal. Twenty-four hours later another fly gave a growth of the same bacillus from its intestine but on its legs nothing but coliform organisms were found. A day later another fly yielded the same bacillus both internally and externally with an addition of a few non-mannite fermenters on its legs. Five days later the last fly gave a culture of the same bacillus together with numerous cocci from its intestine, but no growth was obtained from its legs.

XXIII. On December 15th five flies were put with faeces from Mrs B., from which typhoid bacilli were recovered. Four hours later the faeces were removed and replaced by sugar and water. One fly was examined but the plates were so thickly overgrown that no organisms could be isolated. Two hours later a second fly yielded a number of non-mannite colonies from its intestine but no growth from its legs. Next day a third fly gave no growth from its legs or intestine. Two days later the last two flies were killed. From their legs only cocci were recovered while from the intestine there was a growth of cocci and non-mannite fermenters.

XXIV. This experiment was conducted with normal faeces which were inoculated with a twenty hours' broth culture of typhoid bacilli. Only three flies were used; they were allowed access to the faeces for two days at the end of which time they were removed. One fly was then examined. Cultures were only made from the intestine. All the organisms obtained were non-lactose fermenters but $B$. typhosus was not recovered. The chief organism isolated was one that resembled typhoid except that it did not ferment sorbite, produced indol and discoloured litmus milk. A day later the same organism was recovered in large numbers, but always with it a few typhoid colonies were isolated. Two days later a similar result was obtained with the third fly.

\section{Summary and Conclusions.}

1. The chain of evidence incriminating the house fly as a disseminator of typhoid fever is at present fairly complete, but many of the links are weak and not thoroughly strengthened by experimentation.

2. The bulk of experimental work has hitherto been done under highly unnatural and artificial circumstances and the results so obtained cannot be accepted unreservedly as giving a correct view of conditions in nature.

3. The experiments described in the present paper show that flies can ingest typhoid bacilli from natural matter, i.e. human faeces and urine, and carry them for a certain period of time.

4. There is no evidence to show that the typhoid bacilli multiply in the house fly. On the contrary the evidence goes to show that they are not adapted for prolonged life on or in the fly. 
5. It thus follows that the house fly is a purely mechanical carrier of the typhoid bacillus and is not a natural "host" in the strict sense of the term.

6. Many bacilli closely resembling $B$. typhosus in cultural characteristics appear to be natural or, at least, common inhabitants of the intestine of the house fly. These are extremely likely to be mistaken for $B$. typhosus unless the most stringent tests are employed.

7. As might be expected there is evidence to show that a process of bacterial selection occurs in the fly's intestine. Some bacteria appear to flourish but others are rapidly eliminated. Among the latter must be numbered $B$. typhosus.

I have to thank my colleagues at the Lister Institute, particularly Dr Ledingham, Dr Henderson Smith and Dr Petrie, for much valuable help and advice during the course of these investigations. To Prof. Bainbridge, Dr Arkwright, Dr Penfold and Dr Macalister my thanks are also due, inasmuch as they helped to smooth over many difficulties both theoretical and practical.

\section{REFERENCES.}

BaHr, P. H. (1913). Dysentery in Fiji.

Ficker, M. (1903). Typhus und Fliegen. Arch. f. Hygiene, xLvi. 274-283.

Graham-Smith, G. (1911). Further observations on the ways in which artificially infected Flies (Musca domestica and Calliphora erythrocephala) carry and distribute Pathogenic and other bacteria. Report to the Local Government Board on Public Health and Medical Subjects. New Series, No. 53, 31-48.

(1912). An Investigation of the Incidence of the Micro-organisms known as Non-Lactose-Fermenters in flies in normal surroundings and in surroundings associated with Epidemic Diarrhoea. 41st Annual Report of the Local Government Board. Supplement containing the Report of the Medical Officer for 1911-12, Appendix 13, 304-329.

(1913). Flies in Relation to Disease, Non-bloodsucking Flies. Cambridge University Press, pp. 292.

Ledingham, J. C. (1911). On the Survival of Specific Micro-organisms in Pupae and Imagines of Musca domestica raised from experimentally infected larvae. Experiments with B. typhosus. Journ. of Hygiene, XL. 333-340.

Morgan, H. de R. and Ledingham, J. C. G. (1909). The Bacteriology of Summer Diarrhoea. Proc. Royal Soc. Medicine, XI. (Epidem. Sect.), 133-158.

Nicholls, L. (1912). The Transmissions of Pathogenic Micro-organisms by flies in Saint Lucia. Bull. Entomol. Research, III. 81.

Nicoll, W. (1912). On the Varieties of Bacillus coli associated with the House Fly (Musca domestica). Journ. of Hygiene, XI. 381-389.

TebButt, A. H. (1913). On the Influence of the Metamorphosis of Musca domestica upon bacteria administered in the larval stage. Journ. of Hygiene, xII. 516-526. 